\title{
The Role of a New Liquid-Solid Scintillator, a Mixture of DPO, POPOP and Epon 812, for Enhancement of Autoradiograms with Tritium Agents
}

\author{
Toshiyuki NISHIMAKI and Sen-ichi FURUDATE ${ }^{1 * *}$ \\ Departments of Radioisotope Center and 'Laboratory Animal Science, Kitasato University School of Medicine, Sagamihara, Kanagawa \\ 228-8555, Japan
}

(Received 18 November 1997/Accepted 18 February 1998)

\begin{abstract}
It is desirable to shorten expose time in ${ }^{3} \mathrm{H}$-autoradiography. We developed a new solid scintillator, a mixture of DPO, POPOP and Epon 812, for whole-body autoradiography. The new scintillator is originally in a sol form, but quickly transforms to a solid homogeneous film following exposure to room air. The new scintillator enabled whole-body autoradiography to be developed faster when compared with a liquid scintillator or commercially available reagents. We also demonstrated that the new scintillator gave consistently high counting performance and thus shortened duration of exposure when used in whole-body autoradiography, whereas commercially available enhancers were found to be unsuitable for autoradiography, resulting in dappled images. Furthermore the quantitative analyses of the autoradiograms by a densitometer demonstrated the highest density, and ratio with this new solid scintillator among those with a liquid scintillator and commercially available reagents. In conclusion, a new solid scintillator, a mixture of DPO, POPOP and Epon 812, is a useful and cost effective enhancer for the whole-body autoradiography of tritum compounds. — KEY wORDS: autoradiography, enhancer, ${ }^{3} \mathrm{H}$, liquid-solid scintillator, tumor.
\end{abstract}

J. Vet. Med Sci. 60(7): 795-797, 1998

The usefulness of standard autoradiography is limited because of long exposure times usually required for low levels of isotope incorporation. Przybylski [7] first introduced a liquid scintillation cocktail to microscopic autoradiography. The technique increased the efficacy and thus shortened exposure times. Since then, the method has been applied to various samples, and several investigators have reported the advantages of the method over conventional autoradiography [1, 4, 6, 9]. Woodcock and co-workers [10], however, showed the limited role of the liquid scintillation cocktail and suggested that the technique did not significantly increase the silver grain density. Rogers [8] emphasized the importance of the duration of sample drying schedules as one of the factors that give rise to conflicting results. A solid scintillator has recently been used for scintillation counting which retains a similar efficiency to the regular liquid scintillator $[3,5,11]$. The solid scintillator with a high efficiency in the dry state is potentially an excellent material for autoradiography. In the present study, we describe a new scintillator developed in our laboratory. The scintillator is a sol in its original form but quickly transforms to a solid film when used on tissue samples after exposure to room air. We herein demonstrate the efficiency of the new scintillation medium in tissues with ${ }^{3} \mathrm{H}$-labeled compounds.

\section{MATERIALS AND METHODS}

Preparation of the new scintillation medium: A mixture

* Correspondence to: Furudate, S., Department of Laboratory Animal Science, Kitasato University School of Medicine, Sagamihara, Kanagawa 228-8555, Japan. of $1.2 \mathrm{~g}$ of 2,5- diphenyloxazole (DPO), $0.03 \mathrm{~g}$ of 1,4- bis [2- 5- phenyloxazolyl] benzene (POPOP), $150 \mathrm{ml}$ of Triton $\mathrm{X}-100(\% / \mathrm{vol})$ and $150 \mathrm{ml}$ of toluene $(\% / \mathrm{vol})$ was initially prepared. Then $40 \mathrm{~m} l$ of Epon 812 solution (Yuka Shell Epoxy Co., Tokyo, Japan) was later added to the mixture with continuous stirring in a water bath at $80^{\circ} \mathrm{C}$. All chemicals except for Epon 812 were purchased from Wako Pure Chemical Industries (Osaka, Japan).

Autoradiography using X-ray film: A male nude mouse (BALB/c, nu/nu; SLC, Shizuoka, Japan) bearing human prostate LNCaP tumors [2], established at an earlier stage by inoculation of LNCaP cells (American Type Culture Collection, Rockville, U.S.A.), was used for whole-body autoradiography. Suramin, generously supplied by Bayelin Japan, was labeled through an exchange procedure using tritium gas by Amersham International (England). A total of $9,250 \mathrm{KBq}$ of ${ }^{3} \mathrm{H}$-labeled suramin was injected intravenously into the mouse. Six days after the administration of ${ }^{3} \mathrm{H}$-suramin, the mouse was sacrificed using ether anesthesia, frozen rapidly in a dry ice-acetone mixture for $1 \mathrm{~min}$ and then mounted on carboxyl methyl cellulose. The whole-body was sliced sagittally into about $40 \mu \mathrm{m}$ thick sections in deep freezer using a microtome (OT/SEA, Bright Instrument Co., Huntington, England) and dried at $-20^{\circ} \mathrm{C}$ for 5 days. The effects on quality of the image and exposure time of the solid scintillator were compared with the following 4 groups: A) untreated sections, B) sections treated with liquid scintillator, C) sections treated with commercially available reagents "ENHANCE" (NEN Research Products, U.S.A.), and D) sections treated with a solid scintillator. After the samples were completely dried up, each reagent excluding group A was poured and applied, followed by covering with wrap 
films and by covering with Kodak X-ray film (XAR-5, Eastman Kodak Co., Rochester, U.S.A.) at $-80^{\circ} \mathrm{C}$ for either 5 days or 30 days. The films were developed for $3 \mathrm{~min}$ within a Kodak GBX developer at $20^{\circ} \mathrm{C}$, and the quantity was measured with a densitometer (Konica PDA-65, Konica Co., Tokyo).

\section{RESULTS}

Although the new scintillator is a sol state in preparation and in use, it turns into a homogeneous solid form soon after exposure to room air. The new scintillator offers a pure and constant image for whole-body autoradiography (Fig. 1). Whole-body autoradiograms using X-ray films processed by 4 methods are shown in Fig. 2. When the efficiency of the new scintillator was compared with the 3 other groups, the new scintillator had an obviously superior quality of image than those of the other 3 groups with a shorter time of exposure. Silver grains were not detected on the 4th day and appeared only poorly after 30 days exposure (Fig. 2A). The treatment with the liquid

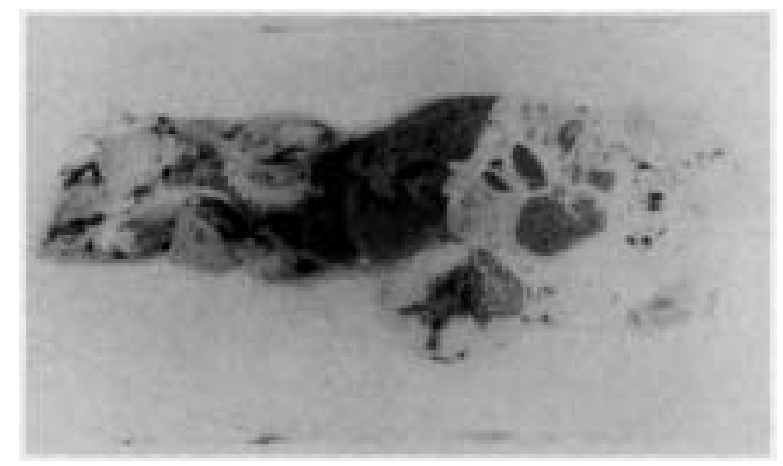

Fig. 1. Application of a new solid scintillator to autoradiography. scintillator gave a slight improvement, and silver grains began to appear at 30 days (Fig. 2B). The commercially supplied "ENHANCE" treatment was better than the liquid scintillator, and the image was clear (Fig. 2C). Using our solid scintillator, the image was much more stronger even when exposed for 5 days (Fig. 2D). It was evident that the silver grains were precise and denser than those treated with "ENHANCE".

The relative grade of intensity of silver grains within the tumor was evaluated and measured quantitatively using a densitometer (Table 1). The counts of density with our new scintillator were found to be the highest among the 4 groups, 26.6 times higher than that of untreated section, and 9.4 times and 5.0 times higher than those of liquid scintillator and "ENHANCE", respectively.

\section{DISCUSSION}

We have reported a solid scintillation holder that enhances the density or count of radioisotopes. It was developed to measure the radioactivity of ${ }^{32} \mathrm{P}$ with a liquid scintillation counter [5]. The method was found to have several advantages, including high counting efficiency and low quenching for colored samples. We have developed a new type of a solid scintillator for enhancing autoradiography with a tritium agent. The scintillator which is in sol form originally, will convert into solid form soon after exposure to room air. In the present study, the new solid scintillator was applied to whole-body autoradiography using ${ }^{3} \mathrm{H}-$ labeled compounds. Our results indicate a consistently higher counting performance when compared with other liquid scintillation methods.

In addition, the results were always reproducible. It should be emphasized that the new scintillator retains its original composition even after it changes into solid form after exposure to room air. Accordingly, we believe that the common problems observed in liquid scintillator

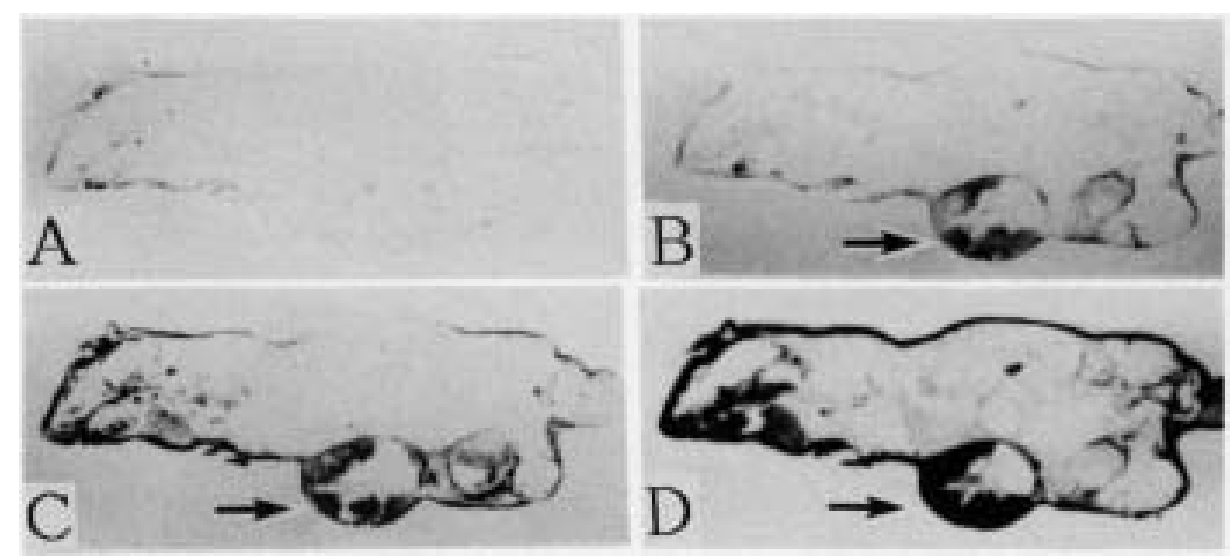

Fig. 2. Whole-body autoradiograms of LNCaP (American Type Culture Collection) xenografts after intravenous administration of ${ }^{3} \mathrm{H}$-labeled anticancer agent. A) Emulsify and exposed for 30 days. B) Emulsify after toluene scintillator and exposed for 30 days (arrow; tumor). C) Emulsify after "ENHANCE" and exposure for 5 days (arrow; tumor). D) Emulsify after solid scintillator and exposure for 5 days (arrow; tumor). 
Table 1. Quantitative analyses of the autoradiogram in Fig. 2

\begin{tabular}{lcccc}
\hline & Untreated & Toluene Scintillator & ENHANCE & Solid scintillator \\
\hline Density & 0.050 & 0.25 & 0.47 & 1.33 \\
Ratio & 1 & 5 & 9.4 & 26.6 \\
\hline
\end{tabular}

Intensities of the tumor region were measured with a densitometer (Konica, PDA-65 type).

autoradiography, occurring during drying schedules of the samples, as previously pointed out by Rogers [8], could be overcome by the application of our new solid scintillator. Yttrium silicate, as a coating on plastic caps or glass fiber filters, is the only solid scintillator that is commercially available at the present time, however its usefulness is limited to scintillation counting, rather than in autoradiography. In addition to the high counting efficiency of our new scintillator in autoradiography, other advantages include the reduction in volumes of disposable waste and in the cost performance. Furthermore, it saves radiation exposure risks to the examiner. Thus, we recommend replacing liquid scintillator autoradiography with the new type of solid scintillator method for autoradiography as a useful tool to study biological and clinical samples.

ACKNOWLEDGMENT. The authors gratefully thank Dr. A. Fujino (Department of Urology, Kitasato University School of Medicine), for his valuable advice.

\section{REFERENCES}

1. Durie, B. G. M. and Salmon, S. E. 1975. High speed scintillation autoradiography. Science 190: 1093-1095.

2. Fujino, A., Furuhata,M., Maru, N., Harano, N., Ao, T., Uchida, T., Koshiba, K., Furudate, S. and Kuwao, S. 1993. Athymic mouse transplantable prostatic tumors with producibility of the specific glycoproteins in human prostate. Focused on LNCaP tumor. Kitasato Med. 23: 194-200.

3. Hawkins, E. F. 1991. Solid scintillators for receptor assays: An environmentally safe alternative to liquid scintillation cocktails. J. Recept. Res. 11: 91-99.

4. Mukherjee, A. S. and Chatterjee, R. N. 1977. Application and efficiency of scintillation autoradiography for drosophila polytene chromosomes. Histochemistry 52: 73-84.

5. Nishimaki, T., Aoki, K., Suzuki, H. and Kashiwagi, N. 1991. Measurement of ${ }^{32} \mathrm{P}$ with solid scintillator holder. Radioisotopes 40: 512-515.

6. Platkowska, E. 1976. Scintillation autoradiography as a quick method for detection of radioactivity in cells and tissues. Bulletin de Lacademie polonaise des sciences. Serie des sciences biologiques CI. VI. 24: 701-704.

7. Przybylski, R. J. 1969. Scintillation radioautography: A new technique designed to augment silver grain number in radioautographies. J. Cell. Biol. 43: 108a.

8. Rogers, A. W. 1981. Scintillation autoradiography at the light microscopic level: a review. Histochem. J. 13: 173-186.

9. Stanulis, B. M., Sheldon, S., Grove, G. L. and Cristofalo, V. J. 1979. Scintillation fluid shortens exposure time in autoradiography. J. Histochem. Cytochem. 27: 1303-1307.

10. Woodcock, C. L. F., Damico-Martel, A., Mclnnis, C. J. and Annunziato, A. T. 1979. How effective is "high-speed" autoradiography. J. Microsco. I17: 417-423.

11. Wunderly, S.W. 1989. Solid scintillation counting: a new technique for measuring radiolabeled compounds. Appl. Radiat. Isot. 40: 569-573. 